\title{
Dental X-rays and Risk of Meningioma
}

Elizabeth B. Claus, MD, PhD, Lisa Calvocoressi, PhD, Melissa L. Bondy, PhD, Joellen M. Schildkraut, PhD., Joseph L. Wiemels, PhD., and Margaret Wrensch, PhD.

Department of Epidemiology and Public Health (EBC, LC), Yale University School of Medicine, New Haven, Connecticut, Department of Epidemiology (MLB), M.D. Anderson Cancer Center, Houston, Texas, Department of Community and Family Medicine (JMS), Duke University School of Medicine, Durham, North Carolina, Departments of Epidemiology and Biostatistics (JW, MW) and Neurological Surgery (MW), University of California at San Francisco School of Medicine, San Francisco, California, and Department of Neurosurgery (EBC), Brigham and Women's Hospital, Boston, Massachusetts

\section{Abstract}

Context-Ionizing radiation is a consistently identified and potentially modifiable risk factor for meningioma, the most frequently reported primary brain tumor in the United States.

Objective-To examine the association between dental x-rays, the most common artificial source of ionizing radiation, and risk of intra-cranial meningioma.

Design and Setting-Population-based case-control study design.

Participants-The study includes 1433 intra-cranial meningioma cases aged 29-79 years diagnosed among residents of the states of Connecticut, Massachusetts, North Carolina, the San Francisco Bay Area and eight Houston, Texas counties between May 1, 2006 and April 28, 2011 and 1350 controls that were frequency-matched on age, sex and geography.

Main Outcome Measure-The association of intra-cranial meningioma diagnosis with selfreport of bitewing, full-mouth, and panorex dental x-rays.

Results-Over a lifetime, cases were more than twice (Odds ratio $(\mathrm{OR})=2.0,95 \%$ confidence interval (CI), 1.4-2.9) as likely as controls to report having ever had a bitewing exam. Regardless of the age at which the films were received, persons who reported receiving bitewing films on a yearly or greater frequency had an elevated risk with odds ratios of 1.4 (95\% CI: 1.0-1.8), 1.6 (95\%CI: 1.2-2.0), 1.9 (95\% CI: 1.4-2.6), and 1.5 (95\%CI: 1.1-2.0) for ages <10, 10-19, 20-49, and $50+$ years, respectively. Increased risk of meningioma was also associated with panorex films taken at a young age or on a yearly or greater frequency with persons reporting receiving such films under the age of 10 years at 4.9 times (95\% CI: 1.8-13.2) increased risk of meningioma. No association was appreciated with location of tumor above or below the tentorium.

Conclusion-Exposure to some dental x-rays performed in the past, when radiation exposure was greater than in the current era, appears to be associated with increased risk of intra-cranial meningioma. As with all sources of artificial ionizing radiation, considered use of this modifiable risk factor may be of benefit to patients.

Address reprint requests to Dr. Elizabeth B. Claus at the Department of Epidemiology and Public Health, Yale University School of Medicine, 60 College St., P.O. Box 208034, New Haven, CT 06520-8034 or at elizabeth.claus@yale.edu.

The authors report no conflict of interest. The authors had full access to all of the data in the study and takes responsibility for the integrity of the data and the accuracy of the data analysis 


\section{Keywords}

cancer; dental x-rays; epidemiology; ionizing radiation; meningioma; computed tomography; population-based; DNA repair genes; risk factors; brain tumor; genetics; diagnostic x-rays

Meningiomas accounted for $33.8 \%$ of all primary brain and central nervous system (CNS) tumors reported in the United States between 2004-2006 and thus represent the most frequently diagnosed primary brain tumor in adults. ${ }^{1}$ Despite this, few studies exist that examine risk factors for this lesion which is frequently associated with neurological complications and decreased quality of life. ${ }^{2}$

The most consistent environmental risk factor identified for meningioma is exposure to ionizing radiation (IR), with relative risks of 6-10 fold reported. ${ }^{3-8}$ However, most studies of IR and meningioma risk include individuals who were exposed to high levels of radiation from sources such as atomic bombs ${ }^{5,6}$ or treatment for oncologic and other medical conditions. ${ }^{3,4}$ Studies that examine risk associated with lower dose exposures more likely to be experienced in the general population are limited in number, include fewer than 200 cases each, and focus on exposure to dental x-rays. ${ }^{9-17}$ To our knowledge no studies have reported on the association between use of computed tomography (CT) and meningioma risk. The studies that report on dental $\mathrm{x}$-ray exposure are suggestive but limited by sample size and the inclusion of cases from time periods with higher dosing regimes than the current era. ${ }^{9-17}$ Several case/control studies in the United States exist, the first which included cases diagnosed between 1980-84 in Los Angeles County reported a significantly increased risk for women associated with a first full-mouth series obtained before age 20 years or before $1945^{15}$ as well as an increased but non-significant risk for men with five or more full-mouth series before $1945 .{ }^{10}$ More recently, Longstreth et al examined 200 cases diagnosed between 1995-98 in Washington State and reported that a history of six or more full-mouth series was associated with increased risk (OR: $2.06,95 \%$ CI: $1.03,4.17)$ but found no evidence for a dose response relationship ( $\mathrm{p}$ for trend $=0.33$ ). ${ }^{12}$ No recent large-scale studies of meningioma risk relative to common ionizing radiation exposure exist, when doses for dental and other procedures have decreased but during which time new radiographic procedures have been introduced, including computed tomography (CT). This report compares the dental and therapeutic radiation histories in 1433 case subjects to those of 1350 control subjects. The large sample size afforded by this population-based study will help to provide a more precise estimate of any association, particularly for the lower exposure levels experienced by more recently diagnosed cases.

\section{MATERIALS AND METHODS}

\section{Study Design}

Eligible case subjects include all persons diagnosed with a histologically confirmed intracranial meningioma among residents of the states of Connecticut, Massachusetts, and North Carolina as well as the Alameda, San Francisco, Contra Costa, Marin, San Mateo, and Santa Clara counties of California and the Brazoria, Fort Bend, Harris, Montgomery, Chambers, Galveston, Liberty, and Waller counties of Texas from May 1, 2006 to April 28, 2011. Cases were identified through the Rapid Case Ascertainment (RCA) systems and state cancer registries of the respective sites and were between the ages of 20 and 79 years at time of diagnosis. Controls were selected by random-digit-dialing by an outside consulting firm (Krieder Research) and were matched to cases by five-year age interval, sex, and state of residence. Study subjects with a previous history of meningioma and/or a brain lesion of unknown outcome were excluded. Subjects were English- or Spanish-speaking. The study, consent forms, and questionnaire were approved by the Human Investigation Committees at 
the Yale University School of Medicine, Brigham and Women's Hospital, the University of California at San Francisco, the M.D. Anderson Cancer Center, and the Duke University School of Medicine. The study was also approved by the State of Connecticut Department of Public Health Human Investigation Committee with some data directly obtained from the CT Tumor Registry in the Connecticut Department of Public Health.

\section{Data Collection}

The physicians of each eligible case were contacted to request permission to approach the case. Cases approved for contact by their physicians and controls identified by Krieder Research were sent an introductory letter. Approximately 1-2 weeks later, a trained interviewer contacted the potential study subject by telephone to administer the interview. Interviews took an average of 52 minutes. Proxies provided information for nine cases and no controls. The questionnaire included detailed questions on demographics, family history of cancer, pregnancy and menstrual history, exogenous hormone history, and medical history including therapeutic and diagnostic radiation procedures. Participants were questioned about the onset, frequency and type of dental care received over their lifetime including orthodontic work, endodontic (root canal) work, dental implants, and dentures. Participants were asked to report the number of times they had received bitewing, fullmouth, or panoramic (panorex) films during four time periods: when aged less than 10 years, 10-19 years, 20-49 years, and 50 years or older. Information was also gathered on the occurrence and timing of therapeutic radiation treatments, specifically radiation or radium treatments to the face, head, neck or chest for both benign and malignant lesions or conditions. Risk factor and screening information was truncated at the date of diagnosis for cases and the date of interview for controls (hereafter referred to as the reference date).

To date, 2228 eligible cases and 2604 eligible controls have been identified. Ninety-eight percent of eligible cases had a consenting physician. Among those cases, $65 \%$ participated in the interview portion of the study while $52 \%$ of eligible controls participated in the interview. Six hundred sixty-six cases were ineligible due to out-of-state residency (45), language (70), recurrent meningioma (83), incarcerated (3), age (50), spinal meningioma (144), pathology unavailable for review (56), mental or medical (i.e. deaf) illness (96), deceased (cause of death other than meningioma) (76), another pathology (i.e. lung metastasis) (16) or other (27). Eighty-five controls were ineligible due to out-of-state residency (6), language (8), a history of previous brain tumor unknown pathology (8), agegroup (1), mental or medical illness (53), deceased (3), or other (8). The sample used in this analysis includes 1433 case and 1350 control subjects.

\section{Statistical Analysis}

The initial portion of the statistical analysis included descriptive statistics. T-tests, chisquare and Fisher exact tests were used to examine the association between the risk of meningioma and independent covariates. To assess the odds of meningioma associated with risk factors, conditional logistic regression was used to provide maximum likelihood estimates of the odds ratios (OR) (adjusted for age, sex, race (white versus non-white), education ( $<=16$ years versus $>16$ years), and history of head CT) with $95 \%$ confidence intervals $(95 \% \mathrm{CI})$ using the statistical package PC-SAS version $9.2 .{ }^{18}$ To avoid attributing the effect of therapeutic ionizing radiation to dental x-rays, persons who received therapeutic radiation to the head, neck, chest, or face were removed from all analyses assessing risk associated with dental $\mathrm{x}$-rays. To assess the association by anatomic location of the meningiomas, we also performed sub-analyses by dividing cases into those with meningiomas located above or below the tentorium as well as those with as skull base tumors using imaging and operative reports. 


\section{RESULTS}

Descriptive statistics are provided in Table 1 . The mean age was 57.5 years for cases versus 57.4 years for controls $(\mathrm{p}=0.74)$. The majority of study subjects were female and White. Cases and controls did not differ by age, race, sex, and geographic location. Controls were more likely to have 16 or more years of schooling and to have a salary greater than $\$ 75,000$.

\section{Dental X-Rays}

Table 2 compares reported dental care and imaging histories for cases and controls. All but one control and two cases reported having visited a dentist on at least one occasion although cases were less likely to report seeing a dentist on a yearly basis. Controls reported first seeing a dentist at a younger age than cases ( 8.6 versus 9.6 years, respectively, $\mathrm{p}<0.01$ ). Cases and controls reported no differences in use of orthodontics or endodontics but cases were less likely to report having dentures ( $\mathrm{OR}=0.8,95 \% \mathrm{CI}: 0.6,1.0)$ and more likely to report dental implants ( $\mathrm{OR}=1.3,95 \% \mathrm{CI}: 1.0,1.7)$ relative to controls.

The majority of study subjects reported having had at least one bitewing in their life (95.8\% of cases and $92.2 \%$ of controls) while approximately $75 \%$ of study subjects reported having undergone at least one full-mouth series. Over a lifetime, cases were more than twice as likely as controls to report having ever had a bitewing. Significantly elevated risk is seen across all ages with the exception of persons aged 50 years or older at time of bitewing although the risk estimate for this age group is elevated to a similar extent as for younger ages. Regardless of the age, more frequent receipt of bitewing films was associated with increased risk. A similar (but not statistically significant) elevated risk for meningioma was seen for full-mouth series for persons receiving yearly or more frequent scans at a young age.

As would be expected, the use of panorex films was less frequently reported than for bitewing or full-mouth series (approximately $47 \%$ of study subjects). Significant increases in risk of meningiomas was associated with young age at receipt of screening as well as more frequent screening with persons under the age of 10 years at almost five-fold increase in risk $(\mathrm{OR}=4.9,95 \% \mathrm{CI}: 1.8,13.2)$.

Of note, cases were no more likely to have received a head CT (prior to their diagnosis of meningioma) than were controls (OR=1.0, 95\% CI: $0.8,1.1)$. Very few persons had received a cerebral angiogram (17 cases and 18 controls, $\mathrm{p}=0.7$ ). No association between location of tumor (supra-tentorial versus on or infra-tentorial) and dental x-ray was observed.

\section{Therapeutic Radiation}

One hundred seventy-four participants (114 cases and 60 controls) reported (Table 3 ) having received previous radiation therapy to the head, neck, face, or chest. Cases were more likely to have received such radiation overall (OR: 1.8, 95\%CI: 1.3, 2.5). Cases were more 1.5 (95\% CI: 1.0, 2.2) and $2.8(95 \% \mathrm{CI}: 1.0,7.8)$ times as likely as controls to report receiving radiation for a malignant or benign tumor.

\section{DISCUSSION}

This is the largest case-control study to examine the relationship between dental x-rays and meningioma risk as well as the most recent, allowing for improved examination of the reduced dosing exposure levels over time. Our findings suggest that dental x-rays, particularly when obtained frequently and at a young age, may be associated with an increased risk of intra-cranial meningioma, at least for the dosing received by these study subjects. Earlier analyses based primarily on data drawn from smaller cohorts of patients 
(and who were likely exposed to higher IR doses) also report an increased risk with dental xrays primarily for the higher dose full-mouth series but only when received at high frequency or a young age. ${ }^{10,12,15}$ In their population-based case-control study including 200 cases of meningioma, Longstreth et al (2004) found an association for persons reporting 6 or more full-mouth films ( $\mathrm{OR}=2.06,95 \% \mathrm{CI}$ : 1.03-4.07) but not for persons reporting fewer films nor bite-wing or panorex films. ${ }^{12}$ Preston-Martin et al (1980) reported an increased risk for females who received a full-mouth before the age of 20 years or before 1945 however this was the only type of x-ray examined. ${ }^{15}$ Our findings show statistically significant increased risk with both bitewing and panoramic films. Risk estimates for fullmouth films, although not statistically significant, were consistently in the same direction as for the other two film types. As in Longstreth et al (2004) $)^{12}$ and Preston-Martin et al $(1980)^{15}$ the highest risk for full-mouth was seen in young persons with higher exposure levels. Given the possible error in recall of specific numbers of dental x-rays, we restricted our frequency analyses to yearly or greater versus less than yearly. Of note, the percentages of persons reporting each of the three categories of x-ray in our series matches well to the previous studies.

Strengths to the study include the population-based study design, large sample size (which may have allowed us to detect effects for x-rays with lower effective dose), and relatively consistent magnitude and direction of risk estimates. Histologic confirmation was obtained for all case subjects suggesting that these results may only be applicable to lesions that are deemed in need of surgery rather than conservative management.

Limitations for this study include the possibility of either under- or over-reporting of dental $\mathrm{x}$-rays by study participants. This is a difficult problem in epidemiology as unlike medical care which (at least within cohorts of patients drawn from health maintenance organizations (HMO) or similar) may be confirmed via review of centralized medical records, dental care is generally obtained even for one person from numerous dentists outside of an HMO or hospital-based setting, providing little opportunity for researchers to validate dental reports in a timely or cost-efficient manner. No national database of dental treatment exists within the United States, hence researchers must rely on patient self-report, despite the potential for bias. In the largest $(n=200)$ previous case/control study of dental x-rays and meningioma (Longstreth 2004) researchers validated dental information on 72 cases and 75 controls, estimating that cases and controls saw 6.1 and 6.6 dentists, respectively, over a lifetime. ${ }^{12,19}$ Participants recalled bitewing and panoramic x-rays more accurately than full-mouth series which they over-reported. The extent of the over-reporting was variable by age and was greater for cases for recent visits and greater for controls for visits more distant in time. However, participants recalled $81 \%$ of the dentists visited in their lifetime with the majority of forgotten dentists and dental care procedures involving only 1 or 2 visits. ${ }^{12,19}$ A second validation effort ${ }^{20}$ found that although both cases and controls tended to overestimate the number of dental x-ray visits, recall appeared to be unbiased with measures of agreement between interview and dental chart data similar for cases and controls.

The extent to which risk for meningiomas associated with exposure to ionizing radiation is modified by genotype is a research area of intense interest. Genetic variants in genes involved in the DNA repair pathway, some of which appear common to a number of tumor types, have been implicated in meningioma risk, but not confirmed. ${ }^{21-24}$ Data from Israel provide evidence for genetic predisposition to radiation-associated meningioma, ${ }^{22-24}$ highlighting the role of inherited genetic factors as well as exposure in the development of meningioma. As radiation exposure is in many instances an avoidable exposure, the need to identify high-risk genetic variants is of great importance to potentially decrease meningiomas and likely other tumor risks. Studies such as this one allow for the collection of large numbers of persons with varying gene*environment combinations and hence 
comparison of the effect of exposures such as ionizing radiation across genetic variant; our group plans to further examine these interactions.

The findings presented here are important given that dental $\mathrm{x}$-rays remain the most common artificial source of exposure to ionizing radiation for persons living within the United States. Use of other medical imaging procedures (and hence exposure to ionizing radiation) is on the rise ${ }^{25}$ with the National Council on Radiation Protection and Measurements reporting that the per capita dose of radiation from medical imaging has increased by a factor of approximately six since the early 1980 's. ${ }^{26}$ For the most part these procedures are associated with even higher levels of exposure to IR than are bitewing or full-mouth dental x-rays. These statistics are of note given the primary environmental (and generally modifiable) risk factor consistently identified for meningioma is exposure to ionizing radiation (IR). The American Dental Association's recent statement ${ }^{27}$ on the use of dental radiographs highlights the need for dentists to examine the risk/benefit ratio associated with the use of dental x-rays and confirms that there is little evidence to support the use of dental x-rays "in search of occult pathoses in the asymptomatic patient" or "routine dental radiographs at preset intervals for all patients." Although dental x-rays are an important tool in wellselected patients, efforts to moderate exposure to ionizing radiation to the head is likely to be of benefit to patients and health-care providers alike.

\section{Acknowledgments}

We acknowledge the cooperation of the following Connecticut Hospitals: Bridgeport Hospital, Bristol Hospital, Charlotte Hungerford Hospital, Danbury Hospital, Day-Kimball Hospital, Eastern Connecticut Health Network, Greenwich Hospital, Griffin Hospital, Hartford Hospital, John Dempsey Hospital, Johnson Memorial Hospital, Lawrence Memorial Hospital, Middlesex Hospital, MidState Medical Center, Hospital of Central Connecticut, New Milford Hospital, Norwalk Hospital, St. Francis Hospital and Medical Center, St. Mary's Hospital, Hospital of St. Raphael, St. Vincent's Medical Center, Stamford Hospital, Waterbury Hospital, William Backus Hospital, Windham Hospital, Yale-New Haven Hospital.

This work was supported by NIH R01 grants CA109468, CA109461, CA109745, CA108473, and CA109475 as well as by the Brain Science Foundation and the Meningioma Mommas.

\section{References}

1. CBTRUS. Central Brain Tumor Registry of the United States. Hinsdale, IL: 2010. CBTRUS Statistical Report: Primary Brain and Central Nervous System Tumors Diagnosed in the United States in 2004-2006. website: www.cbtrus.org

2. Wiemels JL, Wrensch M, Claus EB. Epidemiology and Etiology of Meningioma. J Neuro Oncol. 2010; 99(3):307-314.

3. Ron E, Modan B, Boice JD Jr, Alfandary E, Stovall M, Chetrit A, Katz L. Tumors of the brain and nervous system after radiotherapy in childhood. N Engl J Med. 1988; 319(16):1033-9. [PubMed: 3173432]

4. Hijiya N, Hudson MM, Lensing S, et al. Cumulative incidence of secondary neoplasms as a first event after childhood acute lymphoblastic leukemia. JAMA. 2007; 297(11):1207-15. [PubMed: 17374815]

5. Preston DL, Ron E, Yonehara S, Kobuke T, Fujii H, Kishikawa M, et al. Tumors of the nervous system and pituitary gland associated with atomic bomb radiation exposure. JNCI. 2002; 94(20): 1555-63. [PubMed: 12381708]

6. Shintani T, Hayakawa N, Hoshi M, Sumida M, Kurisu K, Oki S, et al. High incidence of meningioma among Hiroshima atomic bomb survivors. J Rad Res. 1999; 40(1):49-57.

7. Sadetzki S, Flint-Richter P, Starinsky S, Novikov I, Lerman Y, Goldman B, Friedman E. Genotyping of patients with sporadic and radiation-associated meningiomas. Cancer Epidemiol Biomarkers Prev. 2005; 14:969-976. [PubMed: 15824172] 
8. Umansky F, Shoshan Y, Rosenthal G, et al. Radiation-Induced Meningioma. Neurosurgical Focus. 2008; 24(5):E7. [PubMed: 18447746]

9. Preston-Martin S. Descriptive epidemiology of primary tumors of the brain, cranial nerves, and cranial meninges in Los Angeles County. Neuroepidemiol. 1989; 8:283-295.

10. Preston-Martin S, Yu MC, Henderson BE, Roberts C. Risk factors for meningiomas in males in Los Angeles County. J Natl Cancer Inst. 1983; 70:863-866. [PubMed: 6573530]

11. Ryan P, Lee MW, North B, McMichael AJ. Amalgam fillings, diagnostic dental X-rays and tumours of the brain and meninges. Eur J Cancer Oral Oncol. 1992; 28B:91-95.

12. Longstreth WT Jr, Phillips LE, Drangsholt MT, Koepsell TD, Custer BS, Gehrels JA, van Belle G. Dental x-rays and the risk of intracranial Meningioma: a population-based case-control study. Cancer. 2004; 100(5):1026-34. [PubMed: 14983499]

13. Preston-Martin S, White SC. Brain and salivary gland tumors related to prior dental radiography: implications for current practice. J Am Dent Assoc. 1990:120151-158.

14. Preston-Martin S, Mack W, Henderson BE. Risk factors for gliomas and meningiomas in males in Los Angeles County. Cancer Research. 1989; 49(21):6137-43. [PubMed: 2790826]

15. Preston-Martin S, Paganini-Hill A, Henderson BE, Pike MC, Wood C. Case/control study of intracranial meningiomas in women in Los Angeles County, California. J Natl Cancer Inst. 1980; 65(1):67-73. [PubMed: 6930521]

16. Preston-Martin S, Henderson BE, Bernstein L. Medical and dental x-rays as risk factors for recently diagnosed tumors of the head. Natl Cancer Inst Monogr. 1985; 69:175-179. [PubMed: 3834328]

17. Rodvall Y, Ahlbom A, Pershagen G, Nylander M, Spannare B. Dental radiography after age 25 years, amalgam fillings and tumours of the central nervous system. Oral Oncol. 1998; 34:265-269. [PubMed: 9813721]

18. SAS Institute Inc.. SAS® 9.2 Macro Language: Reference. Cary, NC: SAS Institute Inc; 2009.

19. Drangsholt M, Baldwin DK, Longstreth WT Jr. Capture-recapture methods to assess lifetime dental radiographic exposure (abstract). J Dent Res. 2003; 82(Spec Iss A):430.

20. Preston-Martin S, Bernstein L, Maldonado AA, Henderson BE, White S. A dental x-ray validation study: comparison of information from patient interviews and dental charts. Am J Epidemiol. 1985; 121:450-9.

21. Bethke L, Murray A, Webb E, et al. Comprehensive analysis of DNA repair gene variants and risk of meningioma. J Natl Cancer Inst. 2008; 100:270-276. [PubMed: 18270339]

22. Rajaraman P, Hutchinson A, Wichner S, Black PM, Fine HA, Loeffler JS, Selker RG, Shapiro WR, Rothman N, Linet MS, Inskip PD. DNA repair gene polymorphisms and risk of adult meningioma, glioma, and acoustic neuroma. Neuro Oncol. 2010; 12(1):37-48. [PubMed: 20150366]

23. Hosking FJ, Feldman D, Bruchim R, Olver B, Lloyd A, Vijayakrishnan J, Flint-Richter P, Broderick P, Houlston RS, Sadetzki S. Search for inherited susceptibility to radiation-associated meningioma by genomewide SNP linkage disequilibrium mapping. Brit J Cancer. 2011; 104(6): 1049-54. [PubMed: 21364586]

24. Flint-Richter P, Sadetzki S. Genetic predisposition for the development of radiation-associated meningioma: an epidemiological study. Lancet Oncol. 2007; 8:403-10. [PubMed: 17466897]

25. Fazel R, Krumholtz HM, Wang Y, Ross JS, Chen J, Ting HH, et al. Exposure to low-dose Ionizing radiation from medical imaging procedures. JAMA. 2009; 361:849-57.

26. Ludlow JB, Davies-Ludlow LE, White SC. Patient risk to common dental radiographic examinations: the impact of 2007 International Commission on Radiological Protection recommendations regarding dose calculation. J Am Dent Assoc. 2008; 139(9):1237-43. [PubMed: 18762634]

27. The Use of Dental Radiographs - Update and Recommendations American Dental Association Council on Scientific Affairs. J Am Dent Assoc. 2006; 137:1304-1312. [PubMed: 16946440] 


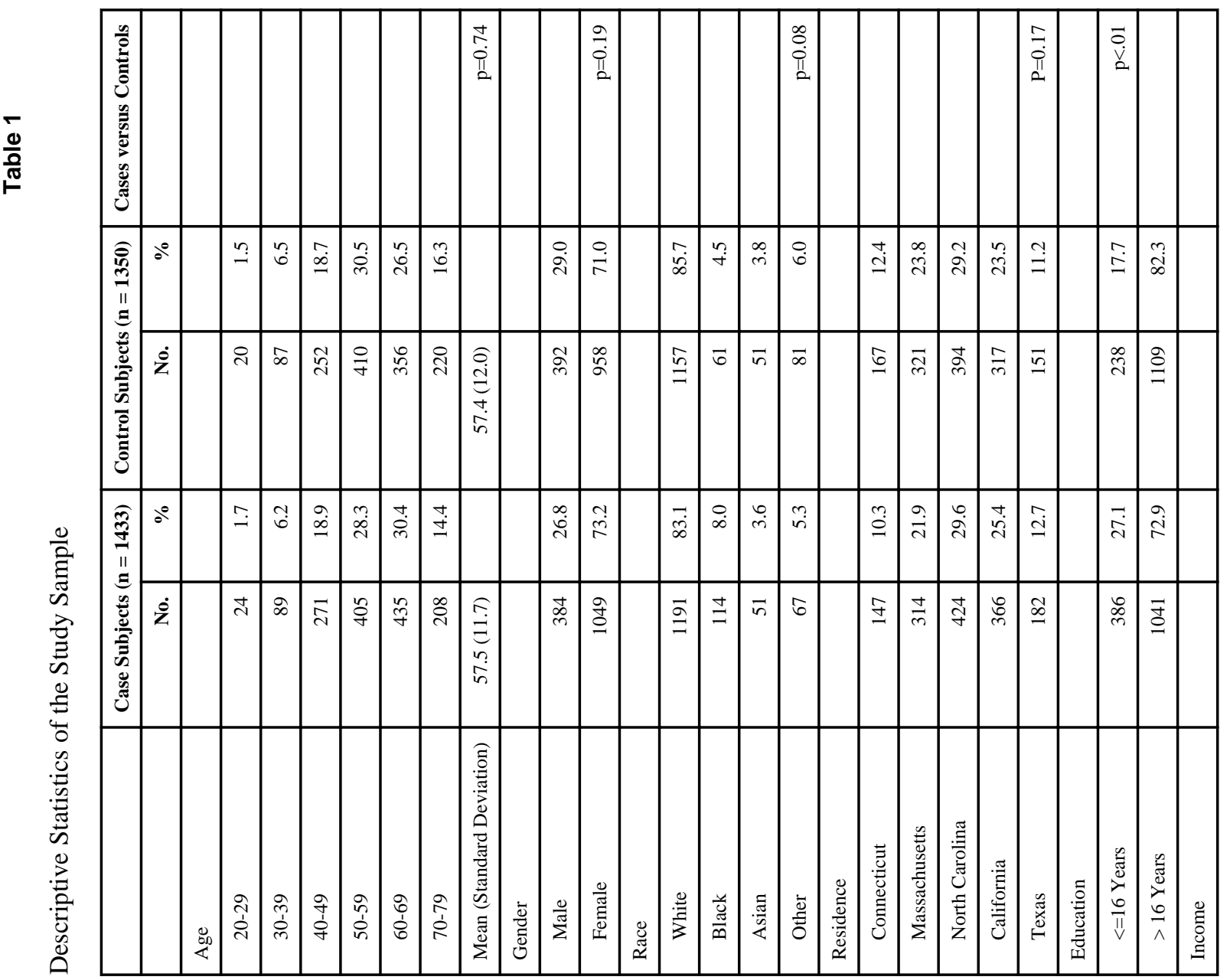




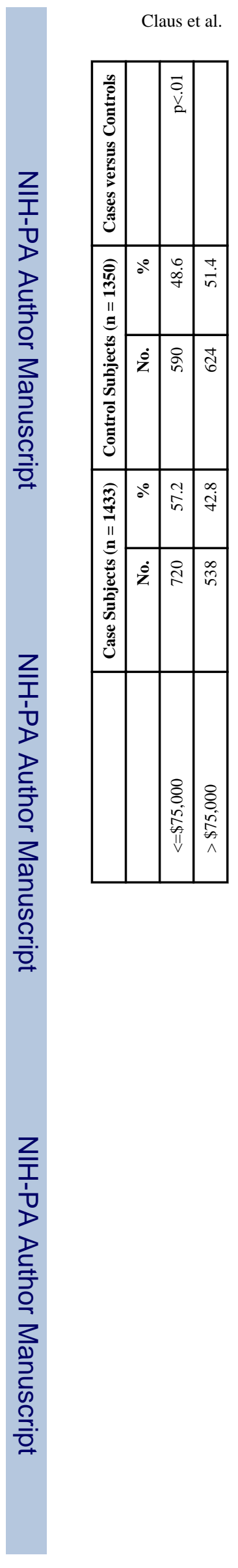

Page 9

Cancer. Author manuscript; available in PMC 2013 September 15. 


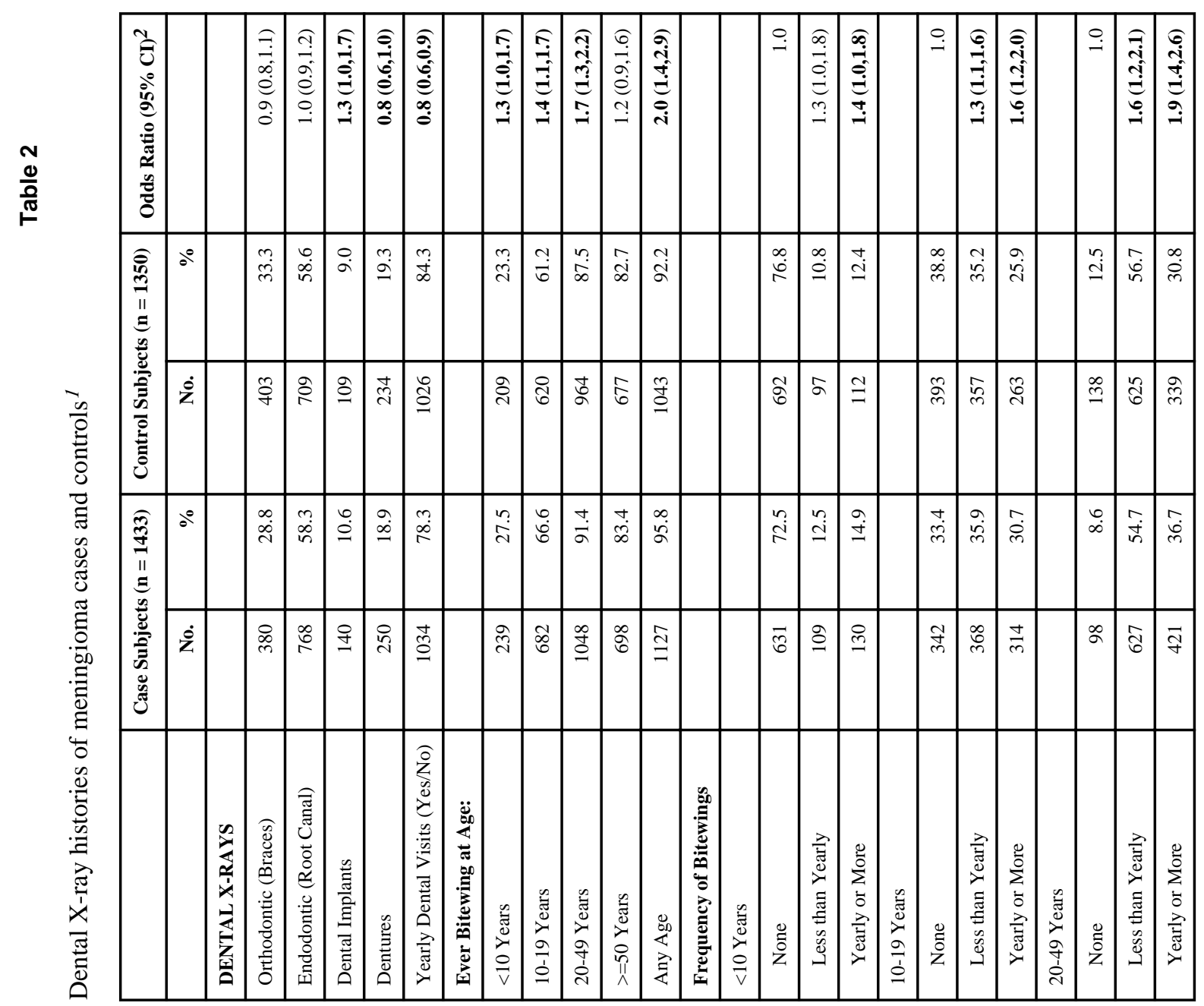

Cancer. Author manuscript; available in PMC 2013 September 15. 


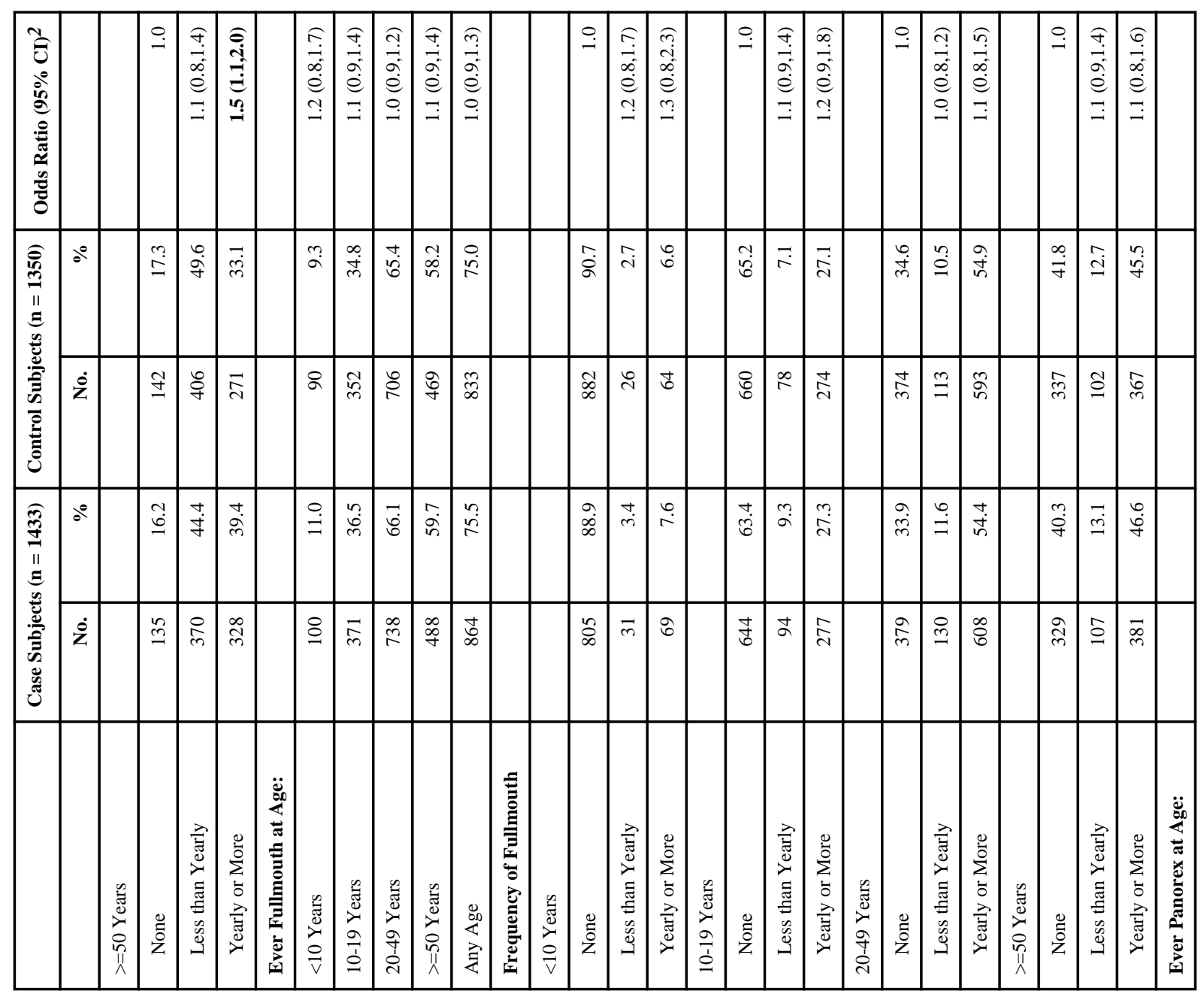




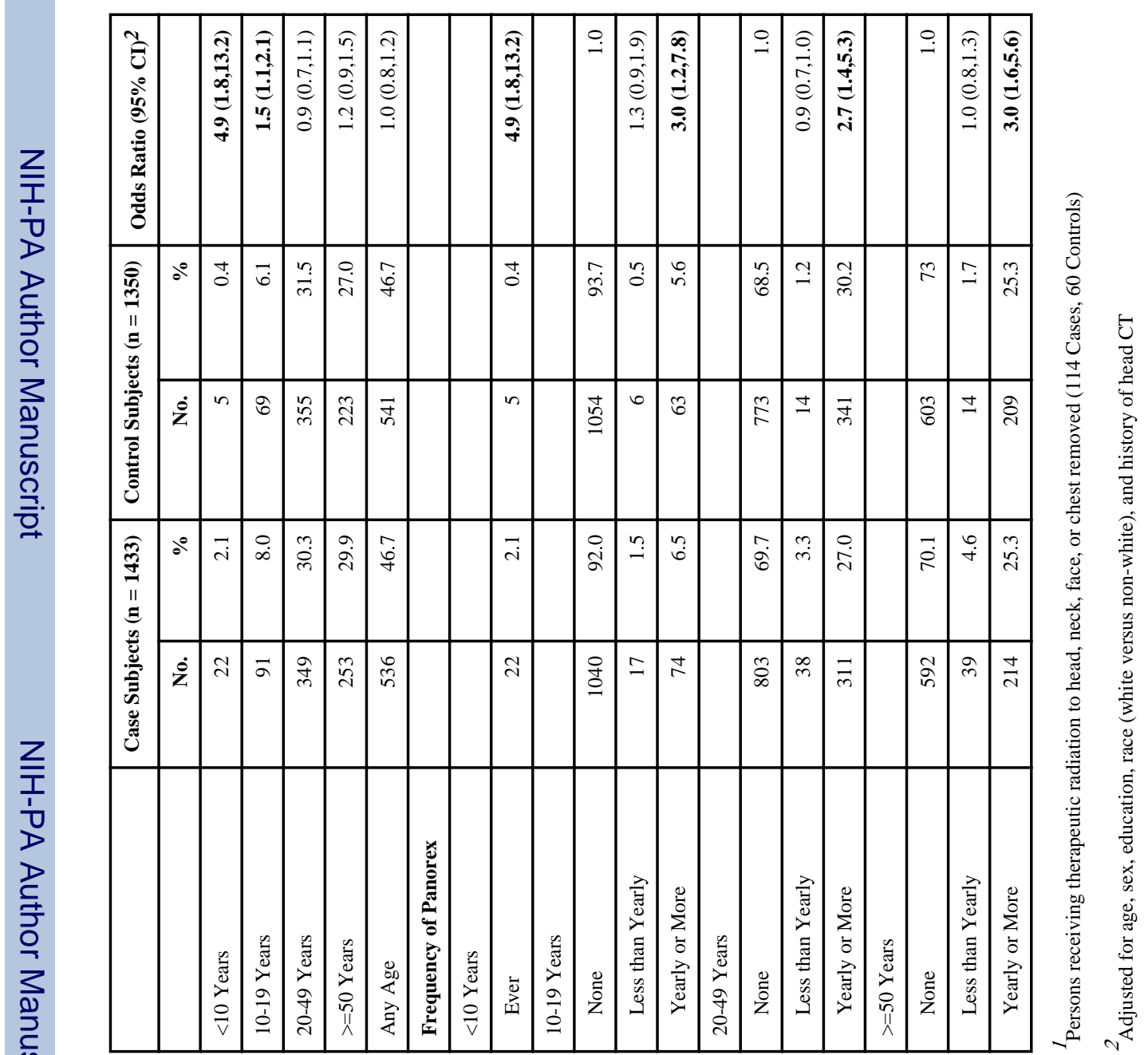




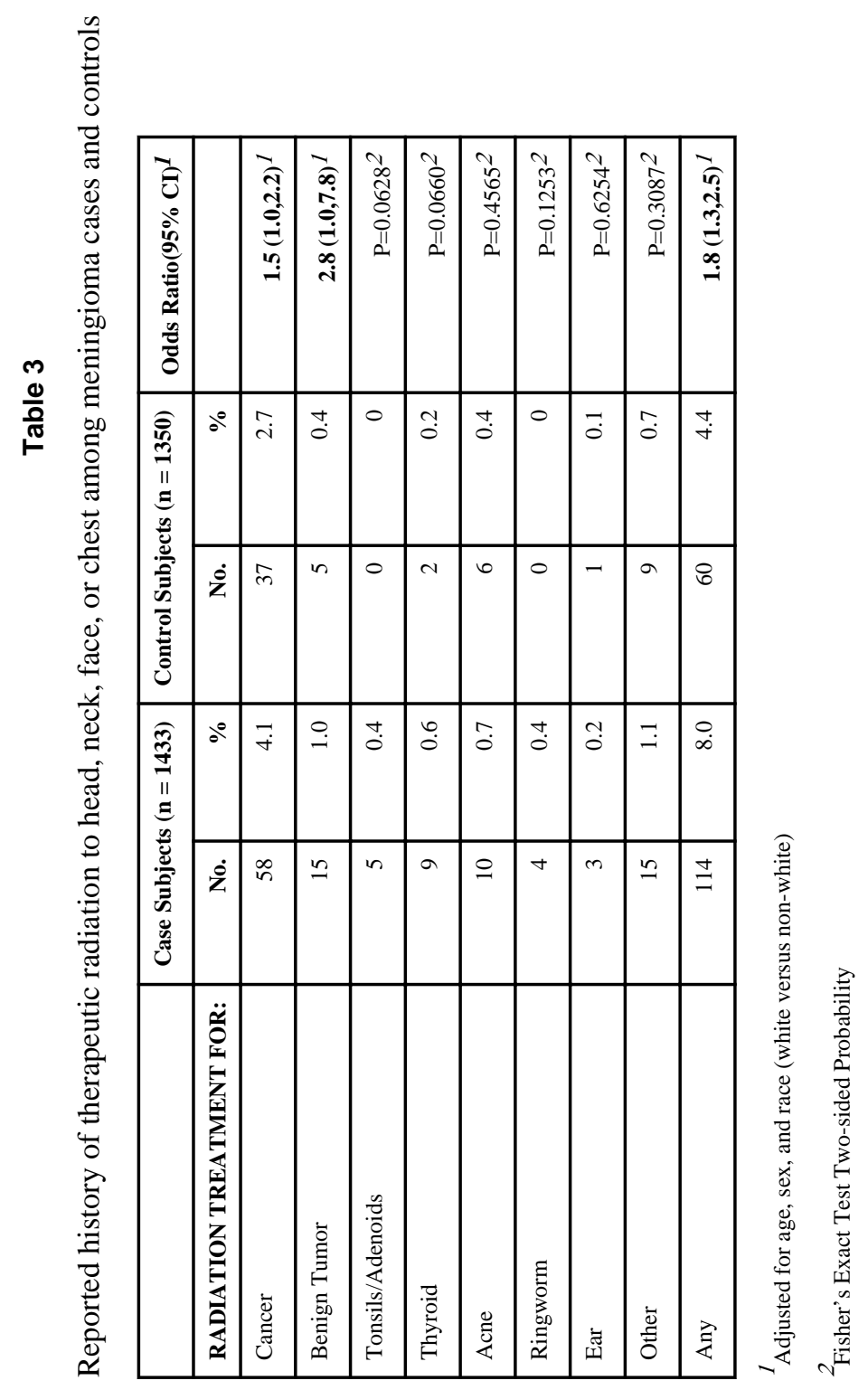

Cancer. Author manuscript; available in PMC 2013 September 15. 